\title{
Measurement and Detectability Limits in Transient Thermography
}

by S. Shepard

Thermal Wave Imaging, Inc., Ferndale, MI USA 48220, sshepard@thermalwave.com

\begin{abstract}
Extraction of a meaningful signal from transient thermography is subject to both intrinsic limitations imposed by thermal diffusion, and extrinsic factors including camera sensitivity, noise, input energy and reflected background radiation. It is possible to define limits of detectability that account for both extrinsic and intrinsic factors. This requires some refinement of the concept of flaw detection, so that the geometric attributes of a flaw (diameter and depth) are treated independently of the thermophysical properties of the constituent materials. We present a predictive model that accommodates a broad range of excitation sources, and facilitates selection of experimental parameters for a given sample configuration.
\end{abstract}

\section{Introduction}

Progress in the practice of thermography for Nondestructive Testing (NDT) has been driven by both advances in the Infrared (IR) camera and personal computer technologies, and the development of physics based signal processing methods. The importance of these seemingly disparate factors is not to be underestimated. The diffusion theory that describes the physics of active thermography was well-established by the late 1960's, when IR cameras first became commercially available. However, those early cameras were inadequate, i.e. far too slow and imprecise, to provide meaningful data that could serve as input to computational methods. Furthermore, the state of computer technology at the time, as well as communication between camera and computer, required tedious digitization or data transfer and computation on a mainframe computer, so that the lag between data acquisition and production of a result could be a matter of hours, if not days. The situation has gradually improved to its current state, where radiometric cameras with high sensitivity, spatial resolution and frame rates, communicate with powerful, inexpensive laptop computers through standardized communication protocols and interfaces. The availability of high-quality experimental data and the ease and speed with which it can be processed has facilitated the development and implementation of modern physics-based signal processing schemes that allow extraction of features that are otherwise undetectable in the raw camera image data, as well as measurement of thermal diffusivity or thickness.

The remarkable progress in the field over the past 40 years has resulted in growing acceptance of thermography as a standalone NDT method. As thermography has become a mainstream NDT tool, it is also subjected to the same requirements and criteria as other accepted methods. One question that inevitably emerges in the process of qualifying thermography for an application involves the detection limits; How deep can a thermography system penetrate a sample or sample type, and what is the smallest feature that can be detected at a given depth in that sample? In practice, the answer that is usually provided by some combination of a) modeling, b) the so-called Rule of Thumb, which states that flaw diameter must be $>\sim 2$, or c) empirical results based on evaluation of a test article representative of the application, or in the best case, a comprehensive Probability of Detection study. While each of these approaches has some utility, none offers much in the way of useful predictive capability that can be applied to an actual system, where knowledge of the system parameters and basic sample properties would provide some ability to estimate whether the system is capable of performing the inspection, and to determine whether a change in system parameters would improve the likelihood of success. There is basically a hierarchy of questions that is implicit in the qualification process:

1. Will a particular thermography system work for a given application?

2. Will modification of the system parameters or components improve or degrade performance significantly?

3. Can ANY thermography system work for this application?

A typical approach to answering these questions is focused on flaw detection. However, in some important respects, that is a secondary issue, as the primary issue is (or should be) to determine whether a thermography system is capable of sensing the presence of an interface (e.g. a back wall of a laminate) at a particular depth. Detection of an interface, a onedimensional problem, is a necessary, but not sufficient, condition for detection of a flaw (a two-dimensional problem). 


\section{Analysis based on camera noise}

An IR thermography system typically comprises an excitation source that heats the sample surface for some time period, and IR camera interfaced to a computer for subsequent processing. Key system parameters include the source energy and duration, the sensitivity frame rate of the camera, and the duration of the acquisition. The camera sensitivity is often specified as the Noise Equivalent Temperature Difference (NETD), which measures the signal variation that occurs when the camera views a temperature-stabilized flat field target. Temperature differences less than the NETD are generally undetectable without additional processing. In most disciplines where a measured signal must be discriminated from noise, it is customary to specify a multiple $(n)$ of the noise level as the minimum signal level for detectability. In practice, the precise determination of $n$ may be the result of a formal Probability of Detection (POD) study, or an ad hoc decision based on the experience of the inspector.

The relationship between signal and noise for a flash thermography system viewing a slab is illustrated in Fig.1, the logarithmic temperature vs. time plot of a point on the surface Noise in the log plot as a horizontal line at temperature no, where $\sigma=N E T D$. In order to detect the presence of the back wall, we require that $\Delta T$ is at least $n$ times greater than $\sigma$, the camera NETD, i.e.

$$
\Delta \text { Twall }=m \sigma
$$

Based on Eq. 1, we can express back wall detectability limit as the condition $m=n$. We also observe that after the cooling process is complete, the sample is in a quasi-equilibrium state, so that the surface temperature indicates the energy absorbed by the sample

$$
Q=\operatorname{mo\rho CL}
$$

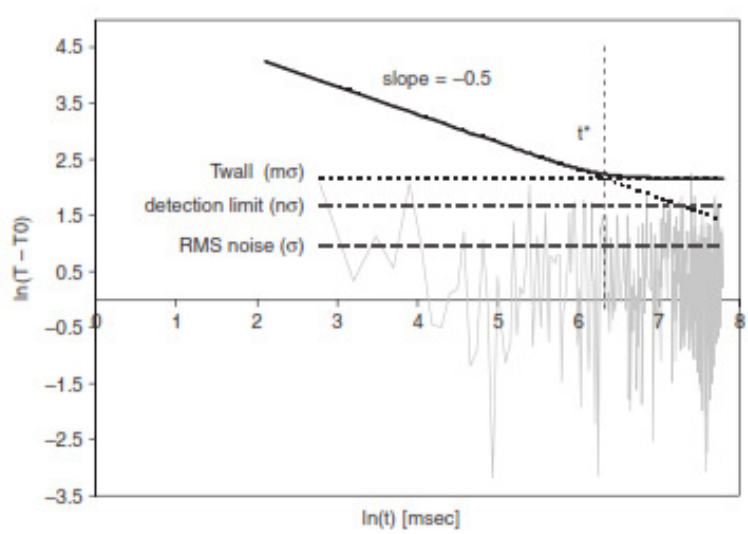

where $\rho$ is the density and $C$ is the specific heat of the sample. The minimum requirement for back wall detectability is $m=n$, so that for a given slab material and thickness, the minimum input energy requirement will be

$$
\mathrm{Q}_{\min }=\mathrm{n} \sigma \rho \mathrm{CL}
$$

Eq. 3 indicates that the camera NETD and the thermophysical properties of the sample define the minimum energy that must be absorbed by the sample for the interface to be detected with a particular system. However, there are additional factors that must also be considered. In particular, the temporal characteristics of the camera must be evaluated relative to 1dimensional diffusion of heat from the surface of an infinitely thick sample. The requirements for these characteristics can be derived from analysis the $2^{\text {nd }}$ derivative of the logarithmic temperature vs. time signal, using the Thermographic Signal Reconstruction Method. These temporal characteristics will be described in detail, leading to a predictive limit for interface detectability, and the relation of that limit to flaw detection. 